Original Paper http://ajol.info/index.php/ijbcs http://indexmedicus.afro.who.int

\title{
Caractérisation de la végétation ligneuse sahélienne du Sénégal: cas du Ferlo
}

\author{
Aissatou Thiam NDONG ${ }^{1,2 *}$, Ousmane NDIAYE ${ }^{1}$, Moustapha Bassimbé SAGNA ${ }^{1}$, \\ Aly DIALLO ${ }^{1}$, Didier GALOP ${ }^{2,3}$ et Aliou GUISSE ${ }^{1,3}$ \\ ${ }^{l}$ Département de Biologie Végétale, Faculté des Sciences et Techniques, Université Cheikh Anta Diop de \\ Dakar, B.P. 5005 Dakar-Fann, Sénégal. \\ ${ }^{2}$ Laboratoire GEODE-UMR 5602-CNRS, Maison de la Recherche de l'Université Jeans Jaurès, 5 Allées \\ Antonio Machado - 31058 TOULOUSE Cedex 1, France. \\ ${ }^{3}$ Observatoire Homme - Milieu (OHM-Téssékéré), Université Cheikh Anta Diop de Dakar, B.P. 5005 Dakar- \\ Fann, Sénégal. \\ *Auteur correspondant, E-mail: assyndong@ @otmail.fr; Tel. (00221) 775405442
}

\section{RÉSUMÉ}

Cette étude vise à évaluer la diversité floristique et la structure du peuplement ligneux de six villages du Ferlo (Keur Momar Sarr, Syer, Mbar toubab, Widou thiengoly, Téssékéré et Labgar). Chaque site a été prospecté puis échantillonné par la méthode des relevés dendrométriques. La richesse spécifique totale est de 38 espèces, elle varie en fonction des sites et est moins importante à Keur Momar Sarr et Syer. Les indices de diversité de Shannon et de régularité évalués n'ont pas trop varié en fonction des sites. Ils sont plus faibles à Téssékéré et plus élevés à Mbar toubab. L'évaluation de la structure horizontale et verticale, montre une prédominance de la strate arbustive. L'Analyse Factorielle des Correspondances a permis de discriminer trois groupements végétaux en étroite relation avec la localisation géographique et la géomorphologie des sites. Ainsi, dans le cadre d'une gestion durable et rationnelle des ressources naturelles en milieu sahélien, cette étude apporte des informations complémentaires sur l'état actuel des peuplements ligneux du Ferlo.

(C) 2015 International Formulae Group. All rights reserved.

Mots clé: Peuplement ligneux, diversité floristique, structure, Ferlo.

\section{Characterization of the Sahelian woody vegetation of Senegal: case of Ferlo}

\section{ABSTRACT}

This study aims to evaluate the floristic diversity and timber stand structure of six villages of Ferlo (Keur Momar Sarr, Syer, Mbar Toubab, Widou Thiengoly, Téssékéré and Labgar). Each site was prospected and sampled by the dendrometric survey method. Total specific richness was 38 species; it varies depending on the site and is less important in Keur Momar Sarr and Syer. Shannon diversity indices and estimated regularity did not vary too much depending on the site. They are lower in Téssékéré and higher in Mbar Toubab. The assessment of horizontal and vertical structure shows a predominance of the shrub layer. The Correspondence Analysis allowed to discriminate three plant groups closely related to the geographical location and the geomorphology of the sites. Thus, within the framework of sustainable and rational management of natural resources in the Sahel, this study provides additional information on the current state of timber stands in Ferlo. (c) 2015 International Formulae Group. All rights reserved.

Keywords: Woody stand, floristic diversity, structure, Ferlo. 


\section{INTRODUCTION}

La région sahélienne en Afrique septentrionale est comprise entre les latitudes $15^{\circ}$ et $20^{\circ} \mathrm{N}$ et les isohyètes 100 et $600 \mathrm{~mm}$, et couvre une superficie de 5,378 millions de $\mathrm{km}^{2}$ (Akpo, 1990). Cette région se singularise du reste de l'Afrique par ses traits physiques et biologiques dont les principales caractéristiques sont l'aridité et la fragilité des écosystèmes.

Au Sénégal, le Sahel correspond à une zone appelée Ferlo ou zone sylvopastorale, située au sud de la vallée (Diallo et al., 2012). L'économie de cette région est basée sur l'exploitation des ressources naturelles, en particulier la végétation. Celle-ci fournit le pâturage naturel pour l'alimentation du bétail et permet aux populations rurales de subvenir à leurs besoins par l'exploitation de divers produits ligneux et non ligneux (Akpo, 1990).

Au cours de ces dernières décennies, ces écosystèmes sahéliens ont été confrontés à de nombreuses sécheresses, à l'accroissement démographique et à l'avancée du front agricole. Cette extension de l'activité agricole fait suite au déclin progressif de l'ancien bassin arachidier des régions historiques du Baol, du Sine, du Saloum et du Kayor (Sarr, 2009).

Il s'en suit une forte dégradation des ressources naturelles liée d'une part à l'aridification caractérisée par une forte variabilité spatio-temporelle de la pluviométrie, et d'autre part aux activités humaines qui accentuent les effets des conditions climatiques, déjà défavorables (Niang, 2009).

Cette dégradation se manifeste par des modifications de la composition floristique et de la structure de la végétation (Bakhoum, 2013). Ce qui peut entraîner une précarisation de plus en plus accentuée des conditions de vie des populations rurales qui sont les plus affectées par les effets de cette dégradation (Diallo et al., 2011a). Cette situation est fort préjudiciable aux conditions de vie des populations et à l'économie de cette zone (Sarr et al., 2013).

Une meilleure gestion des ressources naturelles de cette zone, nécessite la restauration de ces écosystèmes dégradés. Ceci doit reposer sur la connaissance de l'état actuel de ces ressources.

C'est dans ce contexte qu'il faut situer ce travail dont l'objectif principal est d'évaluer et de caractériser la diversité floristique et la structure du peuplement ligneux de six villages du Ferlo. Ce qui permettra d'apporter des informations nécessaires pour la reconstitution et la gestion durable et rationnelle des ressources naturelles végétales de cette région.

\section{MATERIEL ET METHODES \\ Zone d'étude}

D'une superficie qui varie notoirement selon les auteurs de $60000 \mathrm{~km}^{2}$ à $70000 \mathrm{~km}^{2}$ (CSE, 2002), le Ferlo constitue l'une des zones éco-géographiques du Sénégal les plus vastes. Il est «à cheval» aujourd'hui entre les limites administratives des régions de SaintLouis, de Louga et de Matam.

Il appartient, du point de vue climatique, à la zone sahélienne, caractérisée par une alternance d'une longue saison sèche allant d'octobre à juin et une courte saison des pluies entre juillet et septembre. La pluviométrie faible, atteint rarement $300 \mathrm{~mm}$ (Ndiaye, 2013) et est inégalement répartie dans le temps et dans l'espace. Les mois d'août et de septembre qui enregistrent les maxima pluviométriques sont considérés comme le cœur de la saison pluvieuse. Les températures varient toute l'année avec des minima de 15 ${ }^{\circ} \mathrm{C}$ et des maxima de 46 à $48{ }^{\circ} \mathrm{C}$ (Diallo et al., 2011b). L'humidité relative de l'air est très faible, elle est en moyenne $35 \%$ par an. L'évaporation est forte et est de 1800 à 2200 mm par an (Bakhoum, 2013). Deux types de sol ont été distingués (Leprun, 1971):

- les sols sableux du système dunaire constitués de sols brun-rouge subarides neutres à faiblement acides; ils contiennent 80 à $85 \%$ de sable et 3,5\% d'argile en surface. Ils sont pauvres en matières organiques;

- les sols ferrugineux tropicaux, sont de texture sableuse à sablo-argileuse, de couleur rouge, plus ou moins lessivés et pauvres en matières organiques; 
Sur ces sols évolue une végétation de type steppe, caractérisée par un tapis herbacé discontinu et une strate ligneuse clairsemée dominée par Balanites aegyptiaca, Boscia senegalensis et Calotropis procera (Niang, 2009). Le tapis herbacé est composé d'espèces comme Aristida mutabilis, Schoenefeldia gracilis et Cenchrus biflorus (Ndiaye, 2013).

L'élevage constitue l'activité dominante des populations et est de type extensif avec la pratique de la transhumance à une certaine période de l'année (Diallo et al., 2012).

Six villages ont été choisis comme site d'échantillonnage. Il s'agit de Keur Momar Sarr, Syer, Mbar toubab, Widou thiengoly, Téssékéré et Labgar (Figure 1). Leur choix se justifie par l'influence des différents projets, l'installation des observatoires et l'importance des actions anthropiques liées surtout au surpâturage.

\section{Collecte des données}

La méthode de relevé dendrométrique a été utilisée pour cette étude. Nous avons inventorié au total 120 placettes (soit 20 par site), effectués de manière stratifié en tenant en considération la géomorphologie du milieu (plateaux, bas fonds, versants). La technique appliquée consiste à délimiter une placette de $2500 \mathrm{~m}^{2}$ correspondant à l'aire minimale d'échantillonnage au Sahel (Saleh et al., 2013).

Sur chaque placette préalablement délimitée, la liste floristique a été établie et quelques paramètres dendrométriques (hauteur et circonférence des troncs) ont été mesurés sur tous les individus ayant une circonférence à $0,30 \mathrm{~m}$ de sol supérieure ou égale à $10 \mathrm{~cm}$. La détermination floristique est faite grâce à la Flore du Sénégal (Berhaut, 1967). L'actualisation des synonymies a été réalisée à partir de l'énumération des plantes à fleurs d'Afrique tropicale (Lebrun et Stork, 1997).

\section{Traitement des données}

Les données collectées sur le terrain en septembre 2014, ont été soumises à un traitement statistique. La composition et la diversité floristique essentielle pour la caractérisation d'un peuplement (Ramade, 1994) a été mesurée par la richesse spécifique
(S), l'indice de Shannon et Weaver (H'), l'indice de régularité $(\mathrm{R})$ et l'indice de Jaccard.

La richesse spécifique (S) est le nombre total d'espèces de la communauté étudiée. Elle est évaluée pour le peuplement et pour chaque site.

L'indice de Shannon et Weaver prend en compte l'abondance relative des espèces et est calculé par la formule suivante:

$\mathrm{Ni}$ représente le nombre d'individus de l'espèce $\mathrm{i}$ et $\mathrm{N}$ le nombre total d'individus toutes espèces confondues. Un indice de Shannon élevé correspond à une communauté diversifiée (Ndiaye, 2013). Il est associé à l'indice de régularité $(\mathrm{R})$ donné par la relation suivante:

$\mathrm{H}=$ indice de Shannon; $\log _{2}=$ logarithme à base 2 et $\mathrm{S}=$ richesse spécifique totale. $\mathrm{R}$ est minimal (0) quand une seule espèce domine tout le peuplement et maximal (1) lorsque les espèces ont une abondance identique.

L'indice de Jaccard est utilisé pour évaluer la similitude floristique entre les différents sites d'études (Ndong, 2009). Il s'écrit de la façon suivante:

$\mathrm{a}=$ le nombre d'espèces présentes dans les deux stations, $\mathrm{b}$ et $\mathrm{c}=$ les nombres d'espèces absentes d'un des deux stations. La valeur de l'indice sans unité est égale à 0 lorsque les deux sites ne présentent aucune similarité. Par contre, elle est égale à 100, si les deux sites sont totalement identiques.

Ensuite, le traitement des données sur la hauteur et la circonférence du tronc, a permis de réaliser des histogrammes qui illustrent la répartition des individus en fonction de ces paramètres. Cela permet d'établir la distribution ou structure verticale et horizontale des individus dans le but d'apprécier la stratification et le recouvrement du peuplement (Diallo et al., 2012).

Enfin, les 120 relevés ont été traités par l'Analyse Factorielle des Correspondances (AFC). L'AFC permet de traiter les tableaux de contingence et offre la double représentativité des données où espèces et variables sont représentées dans le même espace vectoriel sans déformation (principe de l'équivalence distributionnelle). On 
représente graphiquement les résultats de l'AFC en dressant des nuances de points sur différents axes définissant des plans de projections (Cibois, 2006). Sur ces derniers, s'individualisent des groupes de relevés présentant entre eux plus d'affinités qu'ils n'en ont pour leurs voisins et se trouvent réunis par des espèces plus souvent associées entre elles qu'elles ne le sont avec d'autres.

\section{RESULTATS}

\section{Diversité spécifique}

La richesse spécifique totale est de 38 espèces appartenant à 30 genres et 17 familles (Tableau 1). Le plus grand nombre d'espèce a été recensé à Widou thiengoly (22 espèces).

Les valeurs des indices de diversité de Shannon obtenues n'ont pas trop varié en fonction des sites (Tableau 2). Il est de 2,84 bits pour tous les sites et est plus faible à Téssékéré (2,20 bits) et plus élevé à Mbar toubab (2,50 bits). L'indice de Régularité suit la même tendance. Il est de 0,55 dans toute la zone, plus élevé à Mbar toubab $(0,59)$ et plus faible à Téssékéré $(0,51)$ (Tableau 2). Ceci confirme que le peuplement ligneux est plus diversifié à Mbar toubab et moins diversifié à Téssékéré.

Le Tableau 3 montre le degré de similitude entre les sites appréciés par l'indice de Jaccard. Labgar et Téssékéré sont les sites les plus semblables (64\%), alors que Widou et Keur Momar Sarr sont ceux qui sont moins similaires (23\%). Notons aussi que Syer et Keur Momar (58\%), Labgar et Widou thiengoly (52\%) présentent eux aussi de grandes similitudes. Le nombre important d'espèces communes entre ces sites explique cette ressemblance.

\section{Structure du peuplement}

Distribution des individus selon la hauteur

A l'échelle du peuplement, les hauteurs des individus recensés ont varié de 1,7 à $22 \mathrm{~m}$. Ce qui se traduit ainsi par une distribution asymétrique positive ou asymétrique droite avec prédominance d'individus jeunes (Figure 2).

En fonction des sites, nous avons une distribution asymétrique positive, excepté le site de Keur Momar Sarr où une distribution exponentiellement décroissante, caractéristique des populations en extinction est observée (Figure 2).

\section{Distribution des individus selon la circonférence}

La structure des peuplements, illustrée par la répartition des individus selon la circonférence, montre aussi une distribution asymétrique positive ou asymétrique droite, avec prédominance des jeunes sujets (Figure 3). L'essentiel du peuplement ligneux (96\%) se rencontre dans les classes de circonférence inférieure à $120 \mathrm{~cm}$.

C'est le même constat en fonction des sites. Ces classes, renferment $95 \%$ des individus de Labgar, 96\% des individus de Mbar toubab et $97 \%$ des individus de Keur Momar Sarr, de Syer, de Widou thiengoly et de Téssékéré (Figure 3).

\section{Identification des groupements végétaux}

La matrice de 120 relevés et 38 espèces a été soumise à une Analyse Factorielle des Correspondances (AFC) pour définir les différents groupements végétaux. L'information apportée par les axes factoriels varie de 0,75 à 0,005 soit $19,7 \%$ à $0,12 \%$ (Tableau 4).

Les axes $\mathrm{F} 1$ et $\mathrm{F} 2$ cumulent $32,7 \%$ de l'information. C'est sur ce plan formé par ces deux axes que l'essentiel de notre analyse a été effectué (Figure 4).

En abscisses positives de l'axe F1, nous retrouvons les relevés K3, K6, K10, K15, K16, K20, S7, S11, S13 et S15 qui ont une contribution supérieure à la moyenne $(2,2 \%)$. Ils correspondent respectivement à ceux effectués à Keur Momar Sarr (K1 à K20) et Syer (S1 à S20) situés en bordure du Lac de Guiers. Les espèces se trouvant dans ces abscisses positives et ayant une contribution significative sont Tamarix senegalensis (6,5\%), Salvadora persica (6,9\%), Acacia tortilis var raddiana $(17,6 \%)$ et Leptadenia pyrotechnica $(10,6 \%)$.

Dans les coordonnées négatives de l'axe F1, nous retrouvons les relevés M4, W4, W6, W11, T10, T12, T13, T16 et L19 effectués dans les sites de Mbar toubab (M), Widou (W), Téssékéré (T) et Labgar (L) situés dans la zone des six forages. Sur cette abscisse négative de $\mathrm{F} 1$, les espèces qui ont une meilleure contribution sont : Boscia 
senegalensis $(10,6 \%)$, Calotropis procera $(4,7 \%)$ et Sclerocarya birrea (6\%). Donc l'axe F1 oppose ainsi deux groupes de relevés différents par leur situation géographique et pourrait donc correspondre à un gradient d'humidité.

Dans l'ordonnée positive de F2, nous avons les relevés W4, L2, L14, L16 et L19 effectués dans des bas fonds. Les espèces associées ayant une contribution significative sont: Combretum aculeatum (3,6\%), Combretum nigricans (4,2\%), Feretia apodanthera (12,5\%), Grewia bicolor $(11,2 \%)$ et Dalbergia melanoxylon $(15,2 \%)$.

Dans les coordonnées négatives de F2, nous retrouvons les relevés S5, M4, M17, M18, T1, T10 et T12 associés aux taxons tels que: Acacia raddiana (18,3\%), Calotropis procera $(5,4 \%)$ et Sclerocarya birrea $(5,5 \%)$. Ces relevés correspondent à ceux effectués dans des plateaux. Cet axe oppose aussi deux groupes de relevés différents par leur localisation géomorphologique et pourrait donc correspondre à un gradient topographique.

Ainsi, l'Analyse Factorielle des Correspondances discrimine trois groupements distincts (Figure 4):

- Groupement 1 constitué d'espèces comme Acacia raddiana, Tamarix senegalensis, Prosopis glandulosa et Leptadenia pyrotechnica qui sont déterminantes des sites de Keur Momar Sarr et Syer (situés en bordure du Lac de Guiers).

- Groupement 2 renfermant les espèces comme Balanites aegyptiaca, Combretum micrantum, Combretum glutinosum, Calotropis procera, Sclerocarya birrea et Boscia senegalensis caractéristiques des sites de Mbar toubab, Widou et Téssékéré.

- Groupement 3 composé des espèces comme Dalbergia melanoxylon, Commiphora africana, Grewia bicolor et Acacia pennata, recensées pour la plupart dans le site de Labgar.

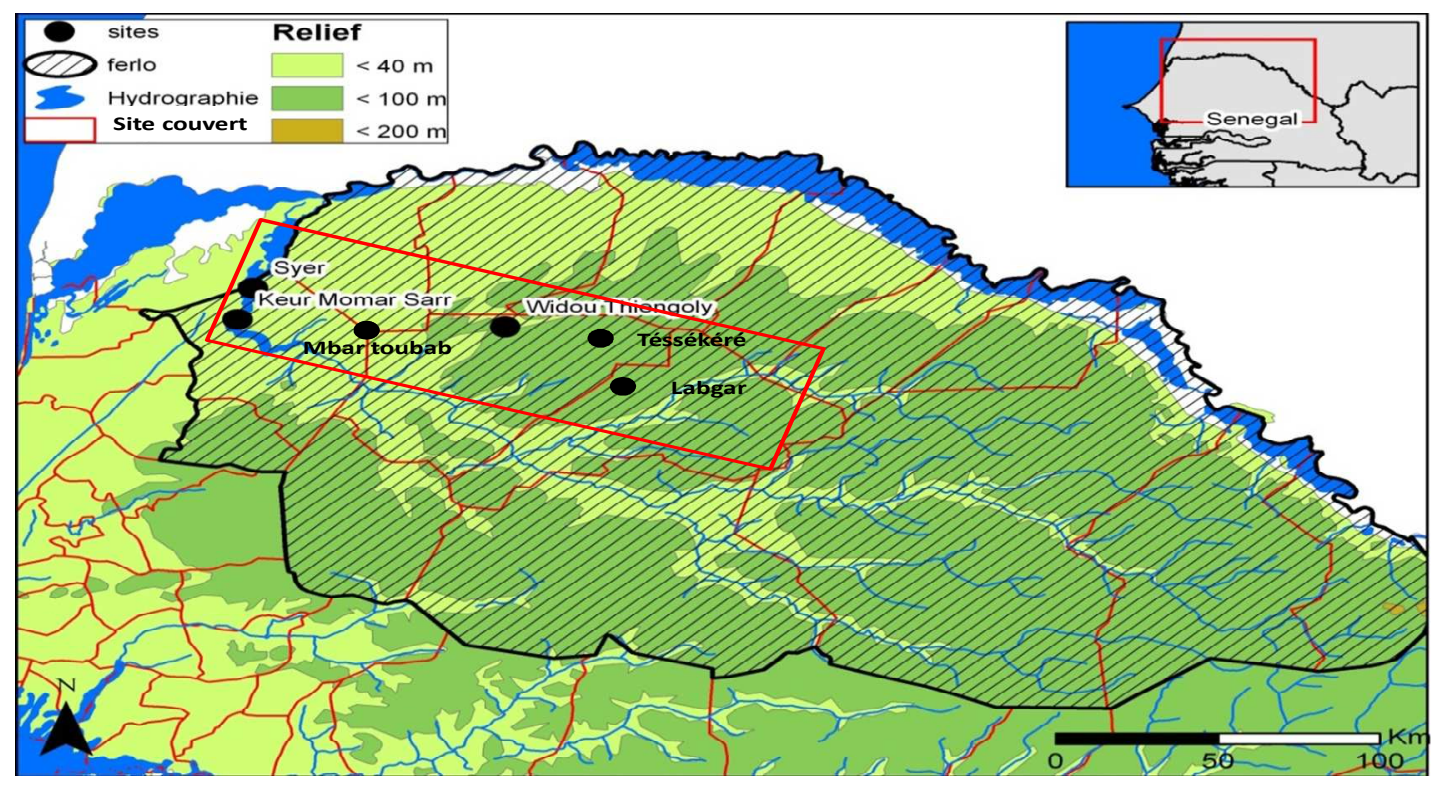

Figure 1: Carte de situation géographique des sites d'études. 

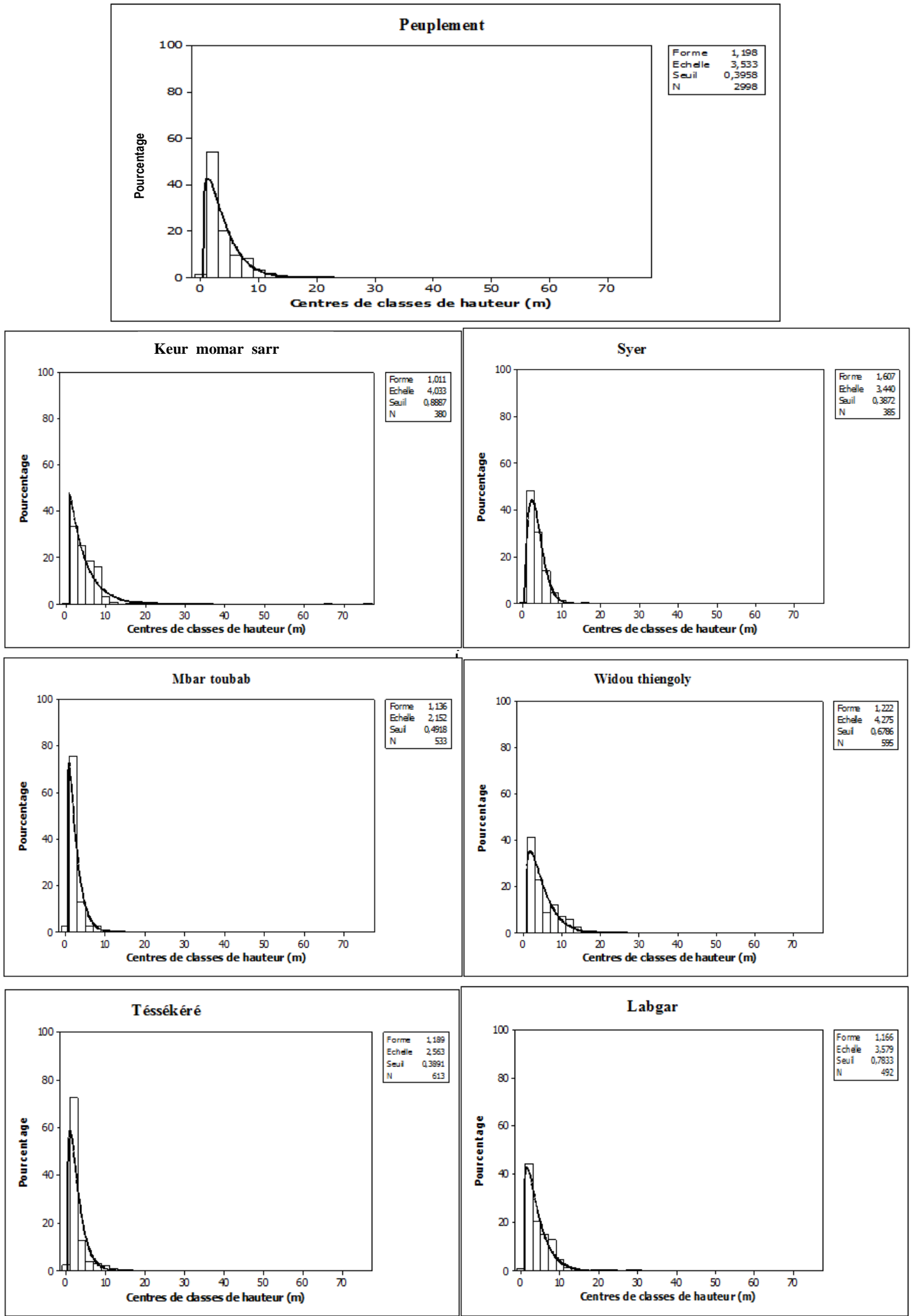

Figure 2: Distribution des individus par classes de hauteur du peuplement et des sites. 

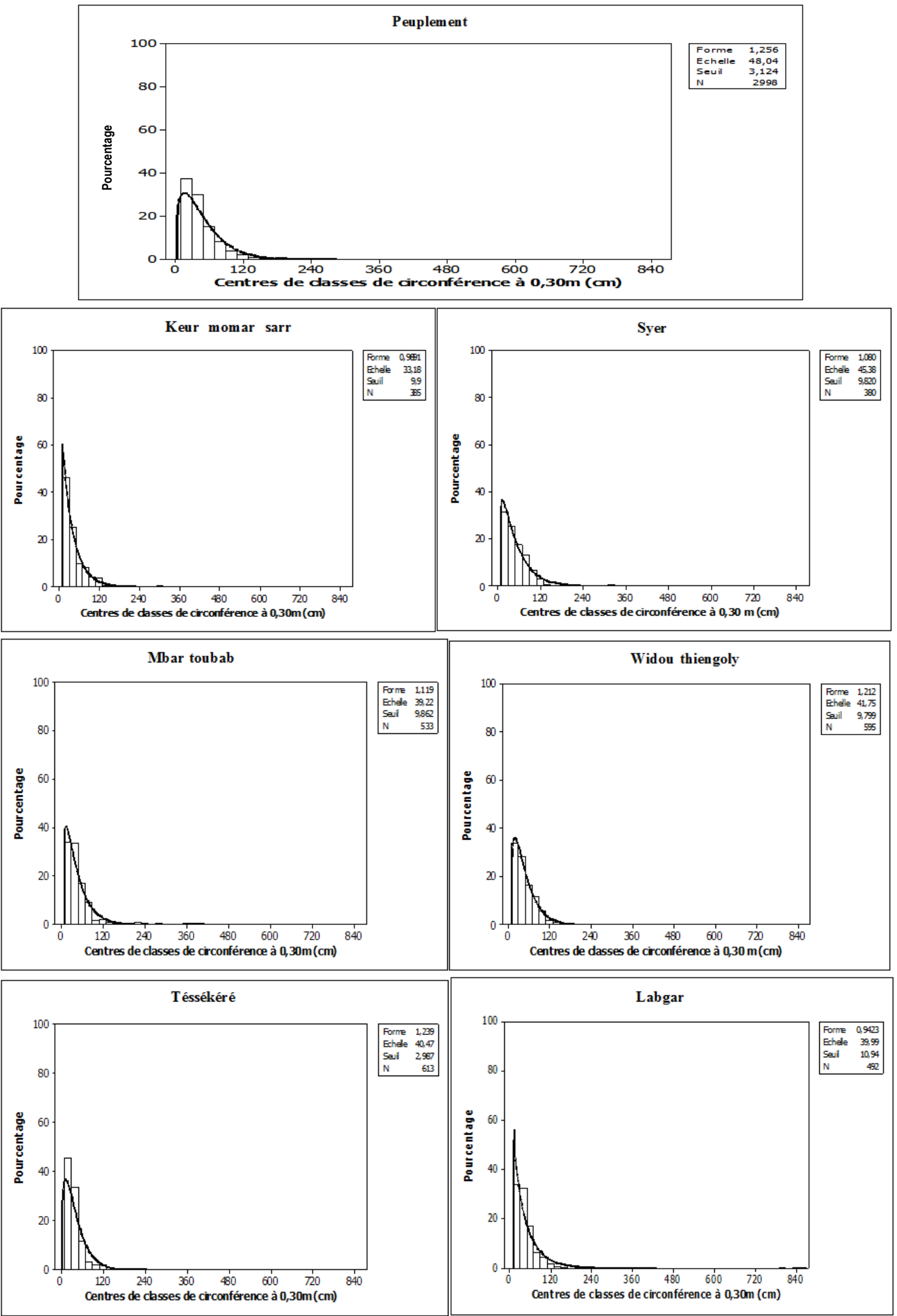

Figure 3: Distribution des individus par classes de circonférence du peuplement et des sites. 
Tableau 1: Liste des espèces ligneuses recensées au Ferlo suivant les sites d'études.

\begin{tabular}{|c|c|c|c|c|c|c|c|}
\hline Familles & 1 & 2 & 3 & 4 & 5 & 6 & Espèces \\
\hline Anacardiaceae & & & + & + & + & + & Sclerocarya birrea (A.Rich.) Hochst. \\
\hline Asclepiadaceae & $\begin{array}{l}+ \\
+\end{array}$ & $\begin{array}{l}+ \\
+ \\
+\end{array}$ & $\begin{array}{l}+ \\
+ \\
+\end{array}$ & $\begin{array}{l}+ \\
+ \\
+\end{array}$ & + & $\begin{array}{l}+ \\
+\end{array}$ & $\begin{array}{l}\text { Leptadenia hastata (Pers.) Decne. } \\
\text { Leptadenia pyrotechnica (Forssk.) Decne. } \\
\text { Calotropis procera (Aiton) W.T.Aiton }\end{array}$ \\
\hline Balanitaceae & + & + & + & + & + & + & Balanites aegyptiaca $(\mathrm{L}$,$) Del$ \\
\hline Bignoniaceae & & & & + & & & Stereospermum kunthianum Cham. \\
\hline Bombacaceae & + & + & $\begin{array}{l}+ \\
+\end{array}$ & + & $\begin{array}{l}+ \\
+\end{array}$ & $\begin{array}{l}+ \\
+\end{array}$ & $\begin{array}{l}\text { Adansonia digitata } \mathrm{L} . \\
\text { Adenium obesum (Forssk.) Roem. \& Schult. }\end{array}$ \\
\hline Burseraceae & & & & + & & + & Commiphora africana (A.Rich.) Engl. \\
\hline Combretaceae & & + & $\begin{array}{l}+ \\
+\end{array}$ & $\begin{array}{l}+ \\
+ \\
+\end{array}$ & + & $\begin{array}{l}+ \\
+ \\
+ \\
+\end{array}$ & $\begin{array}{l}\text { Anogeissus leiocarpus (DC.) Guill. \& Perr. } \\
\text { Combretum aculeatum Vent. } \\
\text { Combretum glutinosum auct. } \\
\text { Combretum micrantum G.Don } \\
\text { Combretum nigricans Lepr. ex Guill. \& Perr. } \\
\text { Guiera senegalensis J.F.Gmel. } \\
\text { Terminalia avicennioides Guill. \& Perr. }\end{array}$ \\
\hline Capparaceae & + & + & + & + & + & + & $\begin{array}{l}\text { Cadaba farinosa Forssk. } \\
\text { Boscia senegalensis (Pers.) Lam. ex Poir. }\end{array}$ \\
\hline Euphorbiaceae & & & $\begin{array}{l}+ \\
+\end{array}$ & & + & & $\begin{array}{l}\text { Jatropha chevalieri Beille } \\
\text { Maerua crassifolia Forssk. }\end{array}$ \\
\hline Fabaceae & $\begin{array}{l}+ \\
+ \\
+\end{array}$ & $\begin{array}{l}+ \\
+ \\
+ \\
+\end{array}$ & $\begin{array}{l}+ \\
+\end{array}$ & $\begin{array}{l}+ \\
+\end{array}$ & $\begin{array}{l}+ \\
+ \\
+ \\
+ \\
+\end{array}$ & $\begin{array}{l}+ \\
+ \\
+ \\
+ \\
+\end{array}$ & $\begin{array}{l}\text { Acacia nilotica (L.) Willd. ex Delile } \\
\text { Acacia_pennata auct. } \\
\text { Acacia tortilis var raddiana } \\
\text { Acacia_senegal (L.) Willd. } \\
\text { Acacia_seyal auct. } \\
\text { Bauhinia rufescens Lam. } \\
\text { Dalbergia melanoxylon Guill. \& Perr. } \\
\text { Faidherbia albida (Delile) A.Chev. } \\
\text { Prosopis glandulosa Torr. }\end{array}$ \\
\hline Menispermaceae & + & + & & + & & & $\begin{array}{l}\text { Cocolus pendulus (J. R. Forst. \& G.Forst.) } \\
\text { Tinospora bakis (A.Rich.) Miers }\end{array}$ \\
\hline Rhamnaceae & & + & + & + & + & + & Ziziphus mauritiana auct. \\
\hline Rubiaceae & & + & & + & & & $\begin{array}{l}\text { Mitragyna inermis (Willd.) K.Schum. } \\
\text { Feretia apodanthera Delile }\end{array}$ \\
\hline Salvadoraceae & + & + & & & & & Salvadora persica $\mathrm{L}$. \\
\hline Sterculiaceae & & & & + & + & & Sterculia setigera Delile \\
\hline $\begin{array}{l}\text { Tamaricaceae } \\
\text { Tiliaceae }\end{array}$ & + & + & & + & + & + & $\begin{array}{l}\text { Tamarix senegalensis DC. } \\
\text { Grewia bicolor Juss. }\end{array}$ \\
\hline
\end{tabular}

(1: Keur Momar Sarr; 2: Syer; 3: Mbar toubab; 4: Widou Thiengoly; 5: Téssékéré; 6: Labgar; +: présence de l'espèce) 
Tableau 2: Diversité floristique des différents sites.

\begin{tabular}{lcccccc}
\hline Paramètres de diversité & $\begin{array}{c}\text { Keur. M. } \\
\text { Sarr }\end{array}$ & Syer & $\begin{array}{c}\text { Mbar } \\
\text { toubab }\end{array}$ & $\begin{array}{c}\text { Widou } \\
\text { thiengoly }\end{array}$ & Téssékéré & Labgar \\
\hline Richesse spécifique totale & 13 & 17 & 19 & 22 & 19 & 19 \\
Richesse spécifique moyenne & 4 & 4 & 5 & 7 & 5 & 5 \\
Indice de Shannon (bits) & 2,21 & 2,23 & 2,5 & 2,35 & 2,20 & 2,42 \\
Indice de Régularité (S.U) & 0,57 & 0,54 & 0,59 & 0,55 & 0,51 & 0,53 \\
\hline
\end{tabular}

Tableau 3: Similitude entre les différents sites échantillonnés du Ferlo.

\begin{tabular}{lccccc}
\hline Sites & $\begin{array}{c}\text { Keur M. } \\
\text { Sarr }\end{array}$ & Syer & $\begin{array}{c}\text { Mbar } \\
\text { toubab }\end{array}$ & Widou thiengoly & Téssékéré \\
\hline Syer & 58 & & & & \\
Mbar toubab & 28 & 33 & & & \\
Widou thiengoly & 23 & 24 & 36 & 46 & \\
Téssékéré & 28 & 37 & 58 & 52 & 64 \\
Labgar & 27 & 30 & 46 & & \\
\hline
\end{tabular}

Tableau 4 : Valeurs propres et inerties des quatre premiers axes de l'AFC.

\begin{tabular}{lcccc}
\hline & F1 & F2 & F3 & F4 \\
\hline Valeur propre & 0,754 & 0,498 & 0,441 & 0,333 \\
\% variance & 19,715 & 13,026 & 11,547 & 8,710 \\
\% cumulé & 19,715 & 32,741 & 44,288 & 52,998 \\
\hline
\end{tabular}




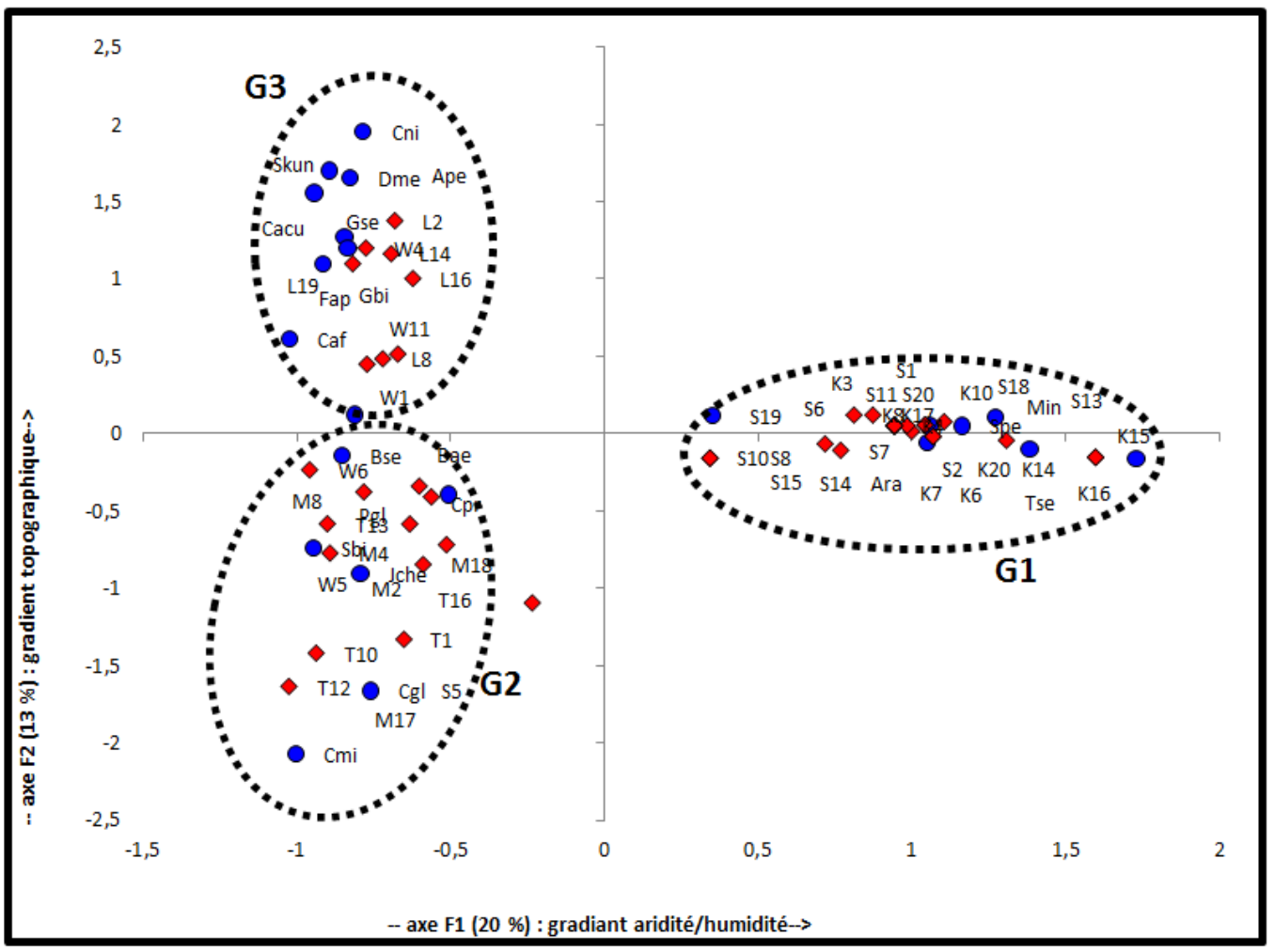

Figure 4: Diagramme de l'AFC: 38 espèces x 120 relevés.

\section{DISCUSSION}

Sur l'ensemble des relevés qui ont été effectués, 38 espèces ont été inventoriées. Ce résultat diffère de celui de Niang (2009), qui a uniquement recensé 20 espèces au Ferlo.

Cette richesse floristique varie en fonction des sites et est moins importante dans les sites situés en bordure du Lac de Guiers. $\mathrm{Ce}$ qui pourrait s'expliquer par le développement intense d'activités de marâichage et d'agriculture sous pluie accompagnés de l'élevage, surtout des petits ruminants dans cette zone.

Balanites aegyptiaca, Leptadenia hastata, Adansonia digitata et Acacia senegal sont retrouvés dans les six sites. Ceci traduit une certaine stabilité et donc une résistance de ces espèces aux aléas climatiques (Bakhoum, 2013). Les espèces Combretum micrantum, Combretum nigricans et Acacia pennata ont été uniquement recensés à Labgar. Faidherbia albida et Maerya cracifolia ne sont rencontrées qu'à Mbar toubab. Par contre, Coculus pendulus et Mitragyna inermis sont uniquement retrouvées à Syer.

L'indice de Shannon mesuré est en moyenne élevé pour tout le peuplement, mais varie selon les sites. Il est plus élevé à Labgar, Widou et Mbar toubab et plus faible à Syer, Keur Momar Sarr et Téssékéré. L'indice de Régularité suit la même tendance. Ceci montre une dominance particulière sur la distribution des individus des différentes espèces à Labgar, Widou et Mbar toubab, mais aussi une stabilité plus grande de ces sites par rapport aux autres sites. Les valeurs d'indice de Jaccard obtenues sont différentes entre les sites, ce qui traduit une différence dans la réponse adaptative des ligneux dans chaque site. Cependant, les valeurs les plus 
élevées sont obtenues entre les sites de Téssékéré et Labgar, de Widou et Téssékéré et de Keur Momar Sarr et Syer. Ce qui traduit une plus grande similarité entre ces sites. D'ailleurs les résultats de l'AFC confirment cette ressemblance floristique.

La structure à l'échelle du peuplement montre une très grande distribution des individus au niveau des premières classes de hauteur et de circonférence. Cela indique que, les strates arbustives sont prédominantes dans la zone. Ceci est dû au fait qu'un peu partout dans la zone, les espèces sont très exploitées par les populations et les herbivores domestiques pendant la saison sèche. Elles sont alors détruites de façon irréversible et les fragiles pâturages surexploités se dégradent (Diallo et al., 2012). C'est ce qui explique la rareté des arbres dans ces peuplements. Cette rareté traduit aussi l'incapacité des arbres à crô̂re normalement en épaisseur suite aux déficits hydriques cumulés (Diallo, 2011). L'ensemble des facteurs précédemment évoqués expliquent l'absence de corrélation entre la croissance en hauteur et en circonférence des individus du peuplement (Ndiaye, 2013).

Trois principaux groupements végétaux, montrant ainsi la disparité qui existe entre quelques localités étudiées, sont identifiés. Ceux-ci ont déjà été signalés dans d'autres travaux (Niang, 2009). Leur disposition fait l'unanimité des auteurs mais on note de petites différences dans la composition floristique à l'intérieur des groupements.

Le groupement à Acacia raddiana est rencontré à Keur Momar Sarr et à Syer. La plupart des espèces qui constituent ce groupement ont été retrouvées dans cette partie du Sahel-Sénégalais par Cornet et Poupon (1977) sur des sols humides de mares ou bas-fonds boisés ou dans les parties les plus basses des inter-dunes du Lac de Guiers. Ce qui prouve leur prépondérance dans des milieux où l'humidité du sol est assez importante. Le groupement à Balanites aegyptiaca est rencontré dans les sites de Mbar toubab, Widou et Téssékéré. Ces espèces à grande amplitude écologique qui le composent, pouvant évoluer sur des sols sableux, pierreux, argileux et alluviaux, indiquent le surpâturage (Bakhoum, 2013) et offrent leur physionomie au peuplement végétal de ces localités. Elles sont mieux adaptées aux conditions climatiques et édaphiques et leur sclérophyllie élevée favoriserait leur développement (Niang, 2009). Le groupement à Dalbergia melanoxylon (Groupement 3) est rencontré uniquement à Labgar. Les espèces qui le composent, se retrouvent souvent sur des surfaces cultivées à très courtes rotations ou sur des jachères. Les défrichements agricoles épargnent généralement ces espèces qui sont les seules bénéficières des ressources édaphiques et se développent pour constituer un peuplement peu diversifiés.

\section{Conclusion}

L'étude a permis d'évaluer la caractéristique structurale du peuplement ligneux de six villages localisé dans la zone sahélienne du Sénégal. Il révèle ainsi, une évolution régressive du peuplement ligneux avec une très forte proportion d'espèces de faible hauteur et circonférence. Cette réduction de la diversité floristique à des répercussions sur les populations locales chez qui l'arbre occupe une place centrale. L'identification des groupements végétaux montre que la répartition de ces espèces ligneuses est conditionnée par les facteurs climatiques et édaphiques.

Dès lors, il sera important d'essayer d'entreprendre des voies et moyens d'assister la régénération de certaines espèces recensées dans cette étude afin de permettre le maintien de ces écosystèmes en dégradation. Il serait aussi intéressant de poursuivre les 
investigations en étudiant la perception populaire sur l'utilisation et la dynamique de ces ressources végétales.

\section{CONFLIT D'INTÉRÊT}

Les auteurs déclarent qu'il n'existe aucun conflit d'intérêt par rapport à cet article.

\section{CONTRIBUTIONS DES AUTEURS}

ATN est le principal investigateur de ce travail et a participé à toutes les phases du travail. $\mathrm{ON}, \mathrm{MBS}$ et $\mathrm{AD}$ ont contribué à la collecte des données sur le terrain et à la rédaction de l'article; DG a été co-encadreur de ce travail et a contribué à la correction de l'article ; AG a été encadreur de ce travail et a contribué à la correction de l'article.

\section{REMERCIEMENTS}

Cette étude a été réalisée grâce à l'appui de Veolia environnement et de l'Observatoire Homme Milieux Téssékéré (OHM).

\section{REFERENCES}

Akpo LE. 1990. Dynamique des systèmes écologiques sahéliens: structure spécifique, productivité et qualité des herbages le forage de Widou thiengoly. DEA en Biologie Végétale, Option Ecologie, FST-UCAD, 65 p.

Bakhoum A. 2013. Dynamique des ressources fourragères: indicateur de résilience des parcours communautaires de Téssékéré au Ferlo (Nord-Sénégal). Thèse de doctorat unique en en Biologie, Productions et Pathologies Animales, Option Ecologie pastorale, FST-UCAD, $115 \mathrm{p}$.

Berhaut J. 1967. Flore Illustrée du Sénégal, (Tome 1) Ed. Clairafrique: Dakar ; 485.

Cibois P. 2006. Principe de l'Analyse Factorielle. 34p. http://cibois.pagesperso orange.fr/PrincipeAnalyseFactorielle.pdf

Cornet A, Poupon H. 1977. Description des facteurs du milieu et de la végétation dans cinq parcelles situées le long d'un gradient climatique en zone sahélienne au Sénégal. Bulletin de l'I.F.A.N.T. 39 sér. A.

CSE (Centre de Suivi Ecologique). 2002. Synthèse des études diagnostiques des sites de l'observatoire du Ferlo. Projet ROSELT/OSS. Rap. Ministère de la jeunesse, de l'environnement et de l'hygiène publique. $10 \mathrm{p}$.

Diallo A, Madiara NF, Ndiaye O, Guissé A. 2011a. Variations de la composition de la végétation herbacée des plantations de Acacia senegal (L.) Willd de la zone de Dahra (Ferlo). Int. J. Biol. Chem. Sci., 5(3): 1250-1264. http://ajol.info/index.php/ijbcs.

Diallo A, Madiara NF, Guissé A. 2011b. Structure des peuplements ligneux dans les plantations d'Acacia senegal (L.) Willd dans la zone de Dahra (Ferlo, Sénégal). Rev. Écol. (Terre Vie), 66: 415427. http://irevues.inist.fr

Diallo A. 2011. Caractérisation de la végétation et des sols dans les plantations de Acacia senegal (L.) Willd dans la zone de Dahra - sud Ferlo sableux. Thèse de doctorat unique en biologie végétale, option écologie, FST-UCAD, 127 p.

Diallo A, Agbangba EC, Thiaw A, Guissé A. 2012. Structure des populations de Acacia senegal (L.) Will dans la zone de Téssékéré (Ferlo nord), Sénégal. Journal of Applied Biosciences, 59: 4297-4306. DOI: http://dx.doi.org/10.4314/jab.v79i1.15.

Lebrun JP, Stork AL. 1997. Enumération des Plantes à Fleurs d'Afrique Tropicale (vol 4). Conservatoires et Jardin Botaniques de la Ville de Genève: 711p.

Leprun JC. 1971. Nouvelles observations sur les formations dunaires sableuses fixées du Ferlo nord occidental (Sénégal). Et. Quaternaires ouest africaines. Bull. Liaison, Sénégal., 31: 69-78. 
Mahamat-Saleh M, Diallo A, Ndiaye O, Madiara NF, Guissé A. 2013. Caractérisation des peuplements ligneux de la zone Cayor Baol (Thiès-Sénégal). Int. J. Biol. Chem. Sci., 7(5): 2117-2132. http://ajol.info/index.php/ijbcs.

Ndiaye O. 2013. Caractéristiques des sols, de la flore et de la végétation du Ferlo, Sénégal. Thèse de doctorat unique en biologie végétale, option écologie, FSTUCAD, $114 \mathrm{p}$.

Ndong A. 2009. Caractérisation biopédologique de la région de Kaolack (zone centre-ouest, Sénégal). Mémoire de
DEA en biologie végétale, FST-UCAD, $60 \mathrm{p}$.

Niang K. 2009. L'arbre dans les parcours communautaires du Ferlo-Nord (Sénégal). Mémoire de DEA en biologie végétale, FST-UCAD, 67 p.

Ramade F. 1994. Eléments d'Écologie Fondamentale $\left(2^{\mathrm{e}}\right.$ édn). Ediscience International ; $579 \mathrm{p}$.

Sarr MA. 2009. Évolution récente du climat et de la végétation au Sénégal (cas du Ferlo), Thèse de doctorat, Université Jean Moulin Lyon 3, LCRE UMR 5600 CNRS, $410 \mathrm{p}$. 